Int. J. Dev. Biol. 57: 773-778 (2013)

doi: $10.1387 / \mathrm{ijdb} .130065 \mathrm{xs}$

\title{
Zfyve9a regulates the proliferation of hepatic cells during zebrafish embryogenesis
}

\author{
NIAN LIU, ZHUO LI, DUANQING PEI and XIAODONG SHU* \\ Key Laboratory of Regenerative Biology, Chinese Academy of Sciences, and Guangdong Provincial Key Laboratory \\ of Stem Cells and Regenerative Medicine, South China Institute for Stem Cell Biology and Regenerative Medicine, \\ Guangzhou Institutes of Biomedicine and Health, Guangzhou, China.
}

\begin{abstract}
Zfyve9 is a FYVE domain protein first identified as a binding partner for SMAD2/3. In vitro studies indicate that it can function either positively or negatively in the TGF- $\beta$ signaling pathway depending on the cell lines used. However, the in vivo function of this protein remains to be investigated. We first analyzed the tissue distribution of zebrafish zfyve9a by in situ hybridization. To investigate the in vivo function of this gene, we performed morpholino mediated loss-of-function assays. We analyzed the expression patterns of liver (cp and fabp10a), pancreas (trypsin and insulin) or gut (fabp2) specific markers to determine whether the formation of these organs is affected by zfyve9a knockdown. We determined the specification of hepatoblast in the zfyve9a morphants (prox1a) and investigated the proliferation and survival of hepatic cells in the morphants by P-H3 staining and TUNEL assay respectively. We report here that zfyve9a is enriched in the zebrafish embryonic liver and required for hepatogenesis. Morpholino mediated knockdown of zfyve9a inhibits the formation of liver by day 4 while the other endoderm-derived organs appear unaffected. We demonstrated that the specification of hepatoblasts is normal in the zfyve9a morphants; however, the proliferation rate of these cells is reduced. Thus, our results reveal the liver-specific function of zfyve9a during early embryogenesis and indicate that the zfyve9a mediated signal is essential for the proliferation of hepatic cells during the expansion of liver bud.
\end{abstract}

KEY WORDS: zfyve9a, liver development, proliferation, zebrafish

\section{Introduction}

The liver is a visceral organ in vertebrates that performs essential roles in metabolism, secretion, detoxification and homeostasis. Structurally, the major components of liver are hepatocytes and bile duct cells and both are derived from the endoderm. Formation of liver during embryogenesis starts with the specification of hepatoblasts from the foregut endoderm which forms the 'liver bud'. These bi-potent hepatoblasts then undergo further growth and differentiate into either the hepatocytes or the bile duct cells (Si-Tayeb et al., 2010). Studies from various models have revealed that the Bmp and Fgf signaling pathways play vital roles during liver specification (Chen et al., 2003, Shin et al., 2007, Zhang et al., 2004) as well as maturation (Berg et al., 2007, Calmont et al., 2006, Jung et al., 1999, Rossi et al., 2001, Sekhon et al., 2004, Shin et al., 2007, Yanai et al., 2008). On the other hand, inhibition of Wnt signaling is required for liver specification(McLin et al., 2007) while the same pathway plays positive roles in later stage of liver development(Micsenyi et al., 2004, Monga et al., 2003, Suksaweang et al., 2004, Tan et al., 2008). Other signaling molecules and transcriptional factors required for liver formation have been identified as well (Chu and Sadler, 2009, Sanchez and Fabregat, 2010, Si-Tayeb et al., 2010, Tao and Peng, 2009).

Zfyve9 (Zinc finger FYVE domain-containing protein 9), also known as SARA (Smad anchor for receptor activation), is a

\footnotetext{
Abbreviations used in this paper: cp, ceruloplasmin; ERBIN, erbb2 interacting protein; gata6, GATA-binding protein 6; hpf, hours post fertilization; fabp2, fatty acid binding protein 2, intestinal; fabp 10a, fatty acid binding protein 10a, liver basic; myca, myelocytomatosis oncogene a; proxla, prospero-related homeobox gene la; P-H3, phospho-histone H3; SMAD2/3, SMAD family member 2/3; TGF- $\beta$, transforming growth factor-beta; TUNEL, terminal deoxynucleotidyl transferase dUTP nick end labeling; Zfyve9, zinc finger FYVE domain-containing protein 9; Zfyve9a, zinc finger, FYVE domain containing 9a.
}

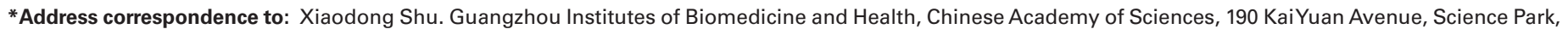
Guangzhou 510530, China. Fax: +86-20-3201-5231. Tel: +86-20-3201-5292. E-mail: shu_xiaodong@gibh.ac.cn

Supplementary Material (two figures) for this paper is available at: http://dx.doi.org/10.1387/ijdb.130065xs

ISSN: Online 1696-3547, Print 0214-6282 
FYVE-type zinc finger containing protein. It was initially identified as a SMAD2/3-binding protein that recruits these SMADs to the TGF- $\beta$ receptors and it is required for the SMAD2-dependent TGF- $\beta$ signaling in a mink cell line (MvlLu) or human mesangial cells (Di Guglielmo et al., 2003, Runyan et al., 2005, Tsukazaki et al., 1998). Recently, ERBIN was identified as a binding partner for both Zfyve9 and SMAD2/3. The binding of Zfyve9 to ERBIN releases the inhibitory effect of ERBIN on SMAD2/3 and induces the SMAD2/3-dependent signaling (Sflomos et al., 2011). However, the exact role of Zfyve 9 in TGF- $\beta$ signaling appears to be contextdependent. For example, it is dispensable for TGF- $\beta$ signaling in COS-7 cells, HeLa cells or B-cell lymphoma cell lines (Bakkebo et al., 2012, Goto et al., 2001). Furthermore, over-expression of Zfyve 9 is able to reduce the TGF- $\beta$ signaling in T cells (Kunzmann et al., 2003). In cultured human kidney epithelial cells, Zfyve9 is required for the maintenance of epithelial cell phenotype through the down-regulation of SMAD2 and up-regulation of mesenchymal markers (Runyan et al., 2009). In Drosophila, Zfyve9 negatively regulates Dpp signaling by recruiting the type 1 serine/threonine protein phosphatase to dephosphorylate the type I TGF- $\beta$ receptor (Bennett and Alphey, 2002). In addition, the Zfyve9 endosomes are involved in the maintenance of Dpp signaling levels across mitosis in the developing wing epithelial cells (Bokel et al., 2006). The in vivo function of Zfyve9 in vertebrates remains to be elucidated.

We identified zebrafish homologs of mammalian zfyve9 gene and analyzed their embryonic expression patterns. We report here that the zfyve9a is a liver-enriched gene and it is required for the proliferation but not specification of hepatoblasts during embryonic liver development.

\section{Results}

\section{The embryonic expression pattern of zfyve9a}

We performed BLAST search and identified two zebrafish homologs of mammalian zfyve9 gene: zfyve9a (Accession \#: XM_001344468, Ensembl(Zv9): ENSDARG00000023701) and zfyve9b (Ensembl(Zv9): ENSDARG00000087295). We first determined the embryonic expression patterns of these genes by whole-mount in situ hybridization. We found that maternal mRNA of zfyve9a was clearly detectable at 2-cell stage (Fig. 1A). Unrestricted low level expression of zfyve9a was observed at gastrulation and somitogenesis stages (Fig. 1 B,C). The expression of zfyve9a then became more restricted and it was highly enriched in liver at day 2 and 3 (Fig. 1 G-J). It was also highly expressed in brain and neural tube at day 3 (Fig. $1 \mathrm{I}-\mathrm{J})$. On the other hand, the staining of zfyve9b was very weak and unrestricted at early stages. Relatively high expression level of zfyve $9 b$ was detected at eyes at day 4 (Supp. Fig. S1). Based on these results, we focused on characterizing the in vivo function of zfyve9a in the development of endoderm-derived organs in this study.

\section{Zfyve9a is required for embryonic liver development}

The hepatic expression of zfyve9a implies that it could be involved in liver formation. We performed morpholino mediated loss-of-function study to test this possibility. We designed a translation blocking morpholino (MO-AUG) which was able to effectively block the translation of mRNA encoding a Zfyve9a-GFP fusion protein (Fig. 2B). We also synthesized a splicing blocking morpholino (MO-SP) targeting the intron2/exon2 junction of the
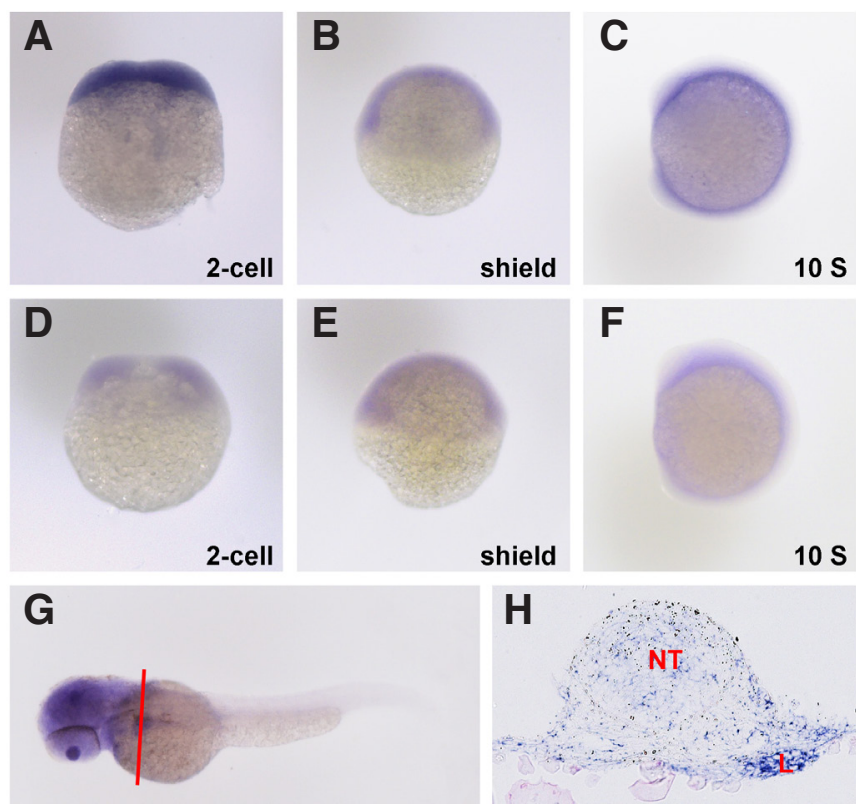

d2
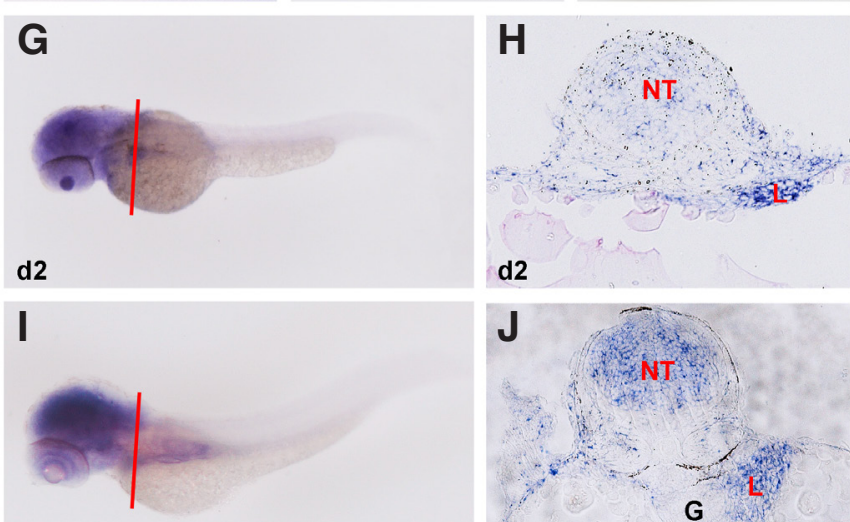

d3

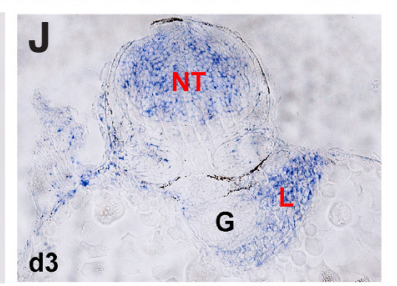

Fig. 1.The embryonic expression pattern of zebrafish zfyve9a. Embryos were hybridized to an antisense probe to zfyve9a (A-C, G-J) or a sense control probe (D-F). (A) Maternal zfyve9a mRNA was detected at 2-cell stage. (B-C) Low level expression of zfyve9a at the shield and 10-somite stages. (G-I) zfyve9a was enriched in the liver at day 2 and 3. It was also highly expressed in the brain and neural tube at day 3. (H) is the cross-section of $(\mathbf{G})$ at the position indicated by the red line. $(\mathbf{J})$ is the cross-section of (I) at the indicated position. NT, neural tube; L, liver; G, gut.

zfyve9a gene which effectively induced the alternative splicing of zfyve9a mRNA as determined by RT-PCR and DNA sequencing (Fig. 2C). We injected embryos with these morpholinos and found the morphants often had mild cardiac edema but otherwise appeared normal (Fig. 2D). We evaluated liver development in these morphants by in situ hybridization using the hepatocyte marker fabp 10a as a probe. As shown in Fig. 2E, both morpholinos severely reduced the expression of fabp 10a at day 3 (25/26 for MO-AUG and 30/32 for MO-SP). The liver defect in the morphants was not a result of general developmental delay since similar defect was obtained at day 4 (46/50 for MO-AUG and 40/43 for MO-SP, Fig. 2F). We further analyzed the expression pattern of $c p$ (ceruloplasmin) which is another marker of hepatocytes and found that the level of $c p$ was similarly down-regulated in the morphants (45/45 for MO-AUG and 42/43 for MO-SP, Fig. 2G). On the other hand, the development of other endoderm derived tissues such as the endocrine pancreatic $\beta$-cell (insulin) (16/16 for MO-AUG and 13/13 for MO-SP, Fig. $2 \mathrm{H}$ ), the exocrine pancreas (trypsin) (23/25 for MO-AUG and 15/18 for MO-SP, Fig. 2I) or the intestine (fabp2) (24/24 for MO-AUG and 12/12 for MO-SP, 
Fig. 2J) was not disrupted in the zfyve9a morphants. Together, these results suggested that zfyve9a is required for the liver but not pancreas or gut development in zebrafish.

\section{Zfyve9a is dispensable for the specification of hepatoblasts}

Liver development in zebrafish begins with the specification of hepatoblasts which form a liver bud at about 30 hours post fertilization (hpf), then these progenitor cells are expanded and differentiated into either hepatocytes or bile duct cells (Chu and Sadler, 2009, Tao and Peng, 2009). The liver defect observed in the zfyve9a morphants could be a result of the failure of either process. We first tested whether the specification of hepatoblasts was affected by the inhibition of zfyve9a. As shown in Fig. 3A, the expression of a pan-endodermal marker foxa3 appeared normal in zfyve9a morphants at $30 \mathrm{hpf}$ (9/9). prox1a is one of the earliest
A

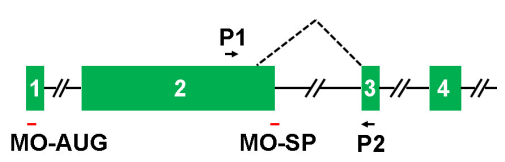

D

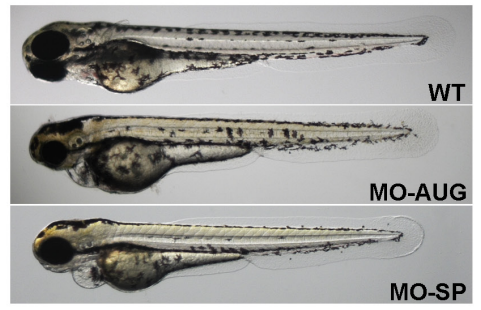

B
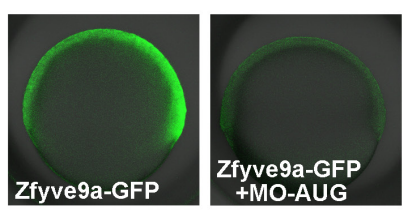

E

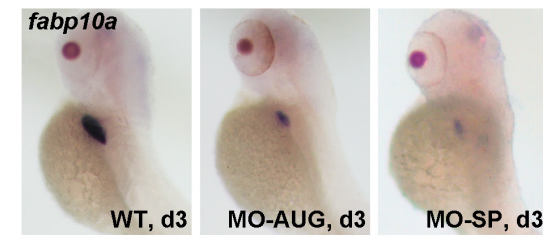

$\mathbf{F}$

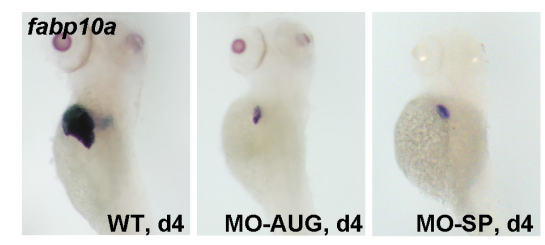

G

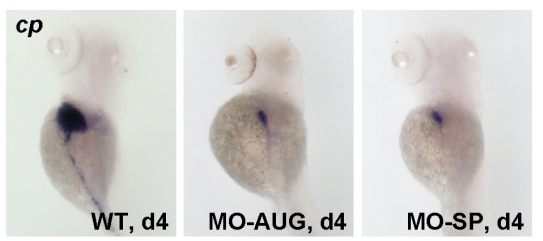

H

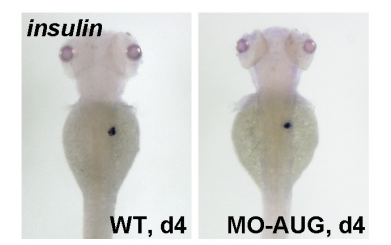

I

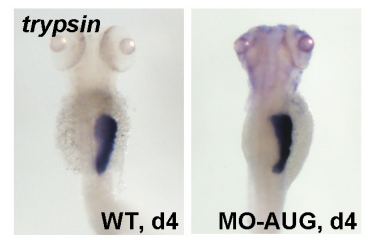

$J$

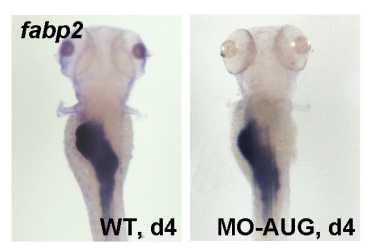

C WT MO-SP

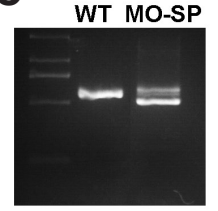

Fig. 2. Zfyve9a is required for liver development. (A) A cartoon of the zfyve9a gene structure shown the positions of MO-AUG, MO-SP and primers used in RT-PCR reaction in (C). (B) MOAUG (4 ng) effectively inhibited the translation of Zfyve9a-GFP mRNA (100 pg) as determined at about 70\% epiboly stage. (C) RT-PCR analysis for the effect of MO-SP. PCR products from wild type and zfyve9a morphants were cloned and sequenced. The lower band in MO-SP treated sample corresponds to a deletion of the C-end of exon 2. (D) Representative live images of wild type embryo and zfyve9a morphants at day 3. (E-F) Expression of fabp10a in WT, MO-AUG or MO-SP injected embryos at day 3 (E) and day 4 (F). (G) Expression of another liver marker, cp, in WT embryos and zfyve9a morphants at day 4. (H-J) Knockdown of zfyve9a did not affect the development of pancreas or gut at day 4. insulin labels the pancreatic beta-cells, trypsin indicates the exocrine pancreas and fabp2 marks the intestine.

liver specific markers and we found that the hepatic expression of this gene was not disrupted in zfyve9a morphants at $30 \mathrm{hpf}$ in the morphants. These results suggest that the specification

We further found that the prox1 expressing area was slightly reduced in zfvye9a morphants at day 2 (9/9, Fig. 3C). Similar result was observed with gata6, which is another pan-endodermal marker (9/9, Fig. 3D). We examined the expression of additional epatic markers ( $m y c a$ and $c p$ ) and both of them were expressed ( specification of hepatoblasts was normal in the morphants;

inhibition of zfyve9a.
insion of liver bud col

\section{Zfyve9a regulates the proliferation but not survival of hepatic cells}

We then investigated whether the proliferation/survival of hepatic cells was affected upon the knockdown of zfyve9a. The gutGFPs854 is a transgenic zebrafish line where the whole endoderm cells are labeled by GFP (Field et al., 2003). The liver cells are clearly distinguishable from other endoderm tissues due to its distinct locations after day 2. We counted the number of GFP-positive hepatic cells and found that it was reduced from $187 \pm 22$ in the control morpholino (MO-CTL) treated embryos $(\mathrm{N}=19)$ to $134 \pm 26$ in the MO-AUG injected embryos $(\mathrm{N}=17)$ at day 2 , which was consistent with our previous in situ hybridization results. We investigated whether the proliferation of hepatic cells was reduced in the morphants by performing whole mount anti-phosphorylated histone $3(\mathrm{P}-\mathrm{H} 3)$ staining. As shown in Fig. 4 A,B, the percentage of $\mathrm{P}-\mathrm{H} 3$ positive hepatic cells was $7.16 \%$ in the MO-CTL treated embryos at $48 \mathrm{hpf}(\mathrm{N}=3546)$. However, this rate was reduced to $2.85 \%$ in zfyve9a morphants $(\mathrm{N}=2144, \mathrm{P}=2.029 \mathrm{E}-06)$. Similar results were observed at $60 \mathrm{hpf}$ (reduced from 6.86\% $(\mathrm{N}=3355)$ to $3.90 \%(\mathrm{~N}=2156), \mathrm{P}=2.7329 \mathrm{E}-07)$. We also performed the terminal deoxynucleotidyl transferase dUTP nick end labeling (TUNEL) assay to determine whether the survival of hepatic cells was affected by zfyve9a knockdown. As shown in Fig. 4 C,D, the ratio of apoptosis in hepatic cells were comparable between the control embryos and zfyve9a morphants at all stages examined ( $P>0.25$ in all cases). These results revealed that the activity of Zfyve9a is required for the proliferation but not survival of hepatic cells.

\section{Discussion}

Zfyve9 is a SMAD2/3 binding protein which has been implicated in the TGF- $\beta$ signaling. Previous 
A

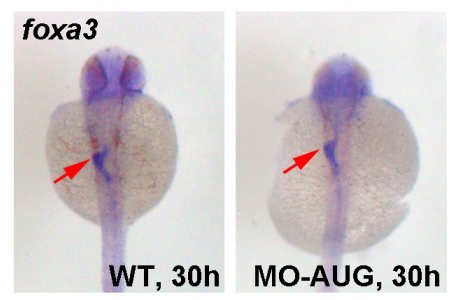

D

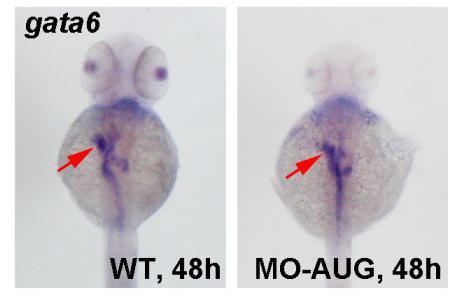

B

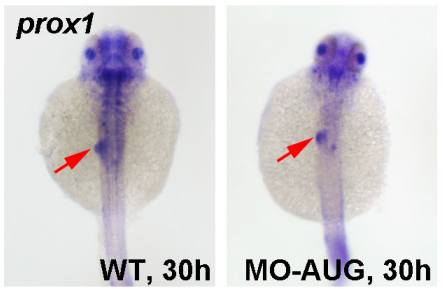

E

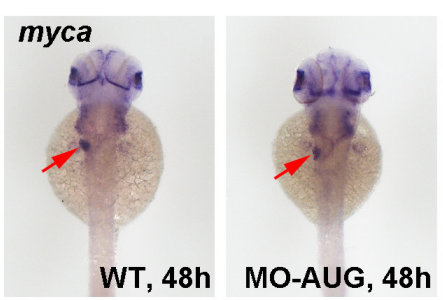

C

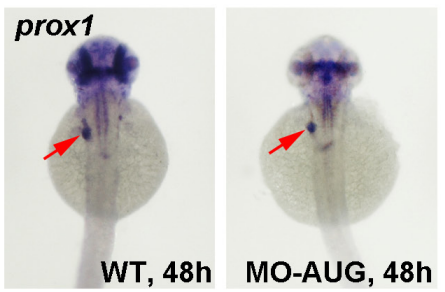

$\mathbf{F}$

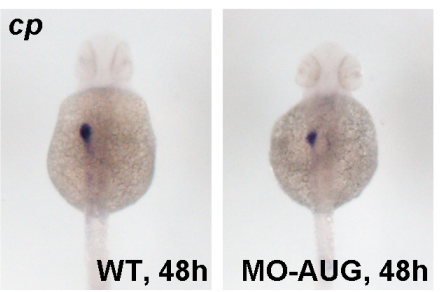

Fig. 3.The specification of hepatoblasts is normal in zfyve9a morphants. (A) The expression of pan-endoderm markerfoxa3 in WT embryos and zfyve9a morphants at $30 \mathrm{hpf}$ (B,C) The expression of prox1a in liver bud was clearly detected in zfyve9a morphants at 30 and 48 hpf. The neuronal expression ofprox1a was severely reduced in morphants at these stages. (D) Expression of gata6 at day 2 in the endoderm of WT embryos and zfyve9a morphants. (E,F) Hepatic expression of myca and $\mathrm{cp}$ were initiated normally in zfyve9a morphants at day 2. Arrows in all panels indicate the liver bud.

studies indicate that it functions either as a positive or a negative regulator of the TGF- $\beta$ pathway in vitro depending on the cell lines used. During the Drosophila wing disc development, it is able to regulate the level of Dpp signaling. However, the in vivo function of this gene in vertebrates remains to be characterized. We identified two homologs of mammalian zfyve 9 gene from the zebrafish genome and found that one of them (zfyve9a) is enriched in the embryonic liver and neuronal tissues. Loss-of-function analysis revealed that the zfyve9a gene is required for the formation of liver but not other endoderm-derived organs such as pancreas and gut. We further found that knockdown of zfyve9a does not affect the specification of hepatoblasts but it is required for the proliferation of these cells during subsequent liver development. A recent study reported that down-regulation of zfyve9 decreases the level of SMAD2 as well as the SMAD2 mediated signaling in a renal epithelial cell line (Runyan et al., 2009). It is interesting to note that haploinsufficiency of both smad2 and smad3 in the smad2+/-; smad3+/- mice reduces the proliferation of hepatocytes and results in liver defect similar to that in the zfyve9a morphants

(Weinstein et al., 2001). It is possible that a defect in the SMAD2/3 signaling is responsible for the liver defect in zfyve9a morphants. In fact, we found that knockdown of zfyve9a in zebrafish embryos reduced the level of phosphorylated-SMAD2 (P-SMAD2, Supp. Fig. S2 A). Furthermore, direct inhibition of the TGF- $\beta$ receptor by small chemical inhibitor Repsox reduced the growth of liver bud (Supp. Fig. S2 B,C). These observations suggest that the Zfyve9a might regulate liver development through the TGF- $\beta$ / SMAD pathway. However, the TGF- $\beta / S M A D$ pathway is essential for early developmental events such as the gastrulation. Injection of an active form of SMAD2 at one-cell stage disrupts the proper gastrulation of the embryo. We were not able to specifically activate this pathway during the expansion of liver bud to rescue the MO-AUG induced liver defects. It remains a challenging task to dissect the late-stage and tissue-specific functions of the TGF- $\beta$ / SMAD signaling during embryogenesis. Further investigations are required to elucidate whether or not zfyve9a can function as a tissue-specific regulator of the TGF- $\beta / S M A D$ pathway during liver development.

A


C

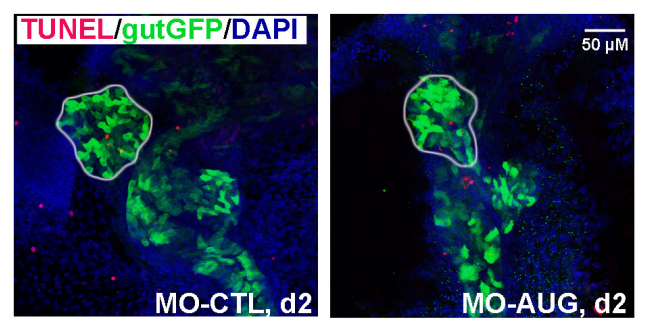

B

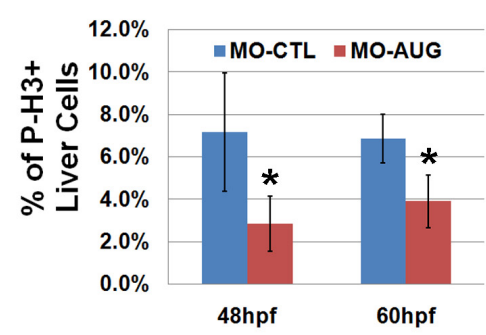

D

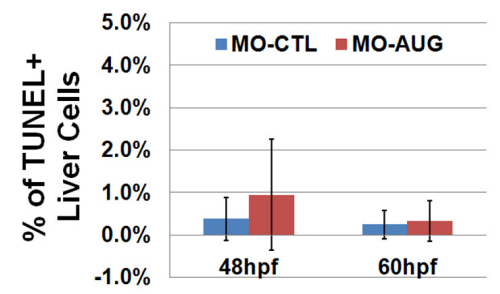

Fig. 4. Zfyve9a regulates the proliferation but not survival of hepatic cells. (A) Representative images of P-H3 staining of embryos at day 2. Green indicates the GFP-labeled endoderm cells. The outlined area is the liver. (B) Statistical results of the percentage of $\mathrm{P}-\mathrm{H} 3$ positive hepatic cells in MO-CTL or MO-AUG injected embryos at 48 and 60 hpf. Stars indicates $P<0.05$ as analyzed by the t-test method. (C) Representative images of TUNEL assay at day 2. (D) The percentage of apoptotic hepatic cells in MO-CTL or MO-AUG treated embryos at 48 and 60 hpf. Error bars in $(B)$ and (D) present the SD. 


\section{Materials and Methods}

\section{Zebrafish manipulation}

Zebrafish adults were bred and embryos were staged using standard protocols as previously described (Westerfield, 1995). Tü and gutGFPs854 lines were used in this study. Zebrafish experiments were approved by the GIBH Institutional Animal Care and Use Committee. Morpholino antisense oligonucleotides to zfyve9a were purchased from Gene-Tools (Corvallis, OR): MO-AUG (CCTCAGCCTGGAAGTAATTCTCCAT, 4 ng/embryo), MO-SP (AATGAACTAGAGACTTTACCTTGCC, 10-12 ng/embryo). The standard control morpholino (CCTCTTACCTCAGTTACAATTTATA, 4 ng/ embryo) was used as the injection control. Morpholinos were injected at 1-cell stage. For testing the efficiency of MO-AUG, embryos were first injected with the mRNA encoding the Zfyve9a-GFP (100 pg) then injected again with the MO-AUG (4 ng/embryo). The expression level of Zfyve9a-GFP was evaluated at the $60-70 \%$ epiboly stage. For validation of the splice-blocking morpholino, the WT or the MO-SP injected embryos were dechorionated at $36 \mathrm{hpf}$ and total RNAs were extracted using the RNAqueous ${ }^{\circledR}-4$ PCR Kit (Ambion, CA). Reverse-transcription was performed using the ReverTra Ace (TOYOBO, Japan). A fragment of zfyve9a was amplified by PCR using the following primers: TGACAGAGGAAAAAGAAATAGAGG (P1) and ACACAAACCCTCGCTTCTTT (P2) and the sequences of PCR products determined by DNA sequencing.

\section{Molecular cloning}

Molecular cloning was performed according to standard protocols. The full length zebrafish zfyve9a gene was cloned by RT-PCR method with the following primers: ATGGAGAATTACTTCCAGGCTGA and TTAAGAGATGATCTCCAGAATGTAGAA. The PCR product was cloned into the pCS2+ (for making mRNA) or the pGM-TEASY (for making probe) vectors. A chimera containing the 1-393 bp of zfyve9a fused to GFP was constructed in the pCS2+ vector (for testing the efficiency of MOAUG). Partial zebrafish zfyve9b gene was cloned by RT-PCR method with the following primers: GAAGGTTTGGTTTGCAGATAATGTC and CCAAAGAGTGACTGAGGTGATTCAG. The PCR product was cloned into the pGM-TEASY (for making probe) vectors. All constructs were confirmed by DNA sequencing. Detailed information about these constructs is available upon request. mRNAs were synthesized using the mMESSAGE mMACHINE Kit (Ambion, CA).

\section{Whole mount in situ hybridization}

Embryos were fixed at the indicated stages and whole-mount in situ hybridization was performed as described (Westerfield, 1995). The sense and anti-sense probes were prepared from the zfyve9a and zfyve9bgenes cloned in the pGM-TEASY vector by in vitro transcription as described (Westerfield, 1995). Other probes used in this study included fabp10a, cp, myca, gata6, prox1a, fabp2, trypsin and insulin.

\section{Proliferation assays}

Whole-mount fluorescence immunostaining was performed as described (Xu etal., 2012). Briefly, embryos were fixed with $3 \%$ formaldehyde in $0.1 \mathrm{M}$ Pipes $/ 1.0 \mathrm{mM} \mathrm{MgSO} / 2 \mathrm{mM}$ EGTA overnight at $4^{\circ} \mathrm{C}$. The yolk was manually removed. Embryos were incubated in acetone for $7 \mathrm{~min}$ at $-20^{\circ} \mathrm{C}$, and then washed with PBST (1X PBS with $0.1 \%$ Tween-20) twice. Embryos were blocked in PBS/4\% BSA/0.3\% Triton X-100 for $1 \mathrm{~h}$ at RT and incubated with a primary antibody (Phospho-Histone H3 (Ser10) (6G3) Mouse mAb (Cell Signaling, 1:100 dilution) or anti-GFP antibody (ab290) (Abcam, 1:1000 dilution)) at $4^{\circ} \mathrm{C}$ overnight. After several washes with PBT ( $0.1 \%$ triton X-100 in PBS), embryos were incubated with a secondary antibody (Alexa Fluor ${ }^{\circledR} 488$ conjugated donkey anti-rabbit IgG or Alexa Fluor®568 conjugated donkey anti-mouse lgG, Molecular Probes, $1: 200)$ for $2 \mathrm{~h}$ at RT. Finally, embryos were washed and counterstained with DAPI $(1 \mu \mathrm{g} / \mathrm{ml}$ in PBS, $5 \mathrm{~min})$ and images were obtained with the Zeiss LSM 710 NLO confocal microscope.

\section{TUNEL assays}

TUNEL assay was performed as described (Curado et al., 2007). Briefly, embryos were fixed as described above. Embryos then embedded in $4 \%$ low melting agarose/1\% gelatin and sectioned with the Leica VT1000S vibratome into $100 \mu \mathrm{m}$ thick slices. Sections were first incubated with the anti-GFP antibody (ab290) (Abcam, 1:1000 dilution), then stained with the In situ Cell Death Detection Kit (TMR red, Roche Diagnostics) according to the manufacturer's protocol. After several washes with PBT (0.1\% triton $\mathrm{X}-100$ in PBS), embryos were incubated with a secondary antibody (Alexa Fluor $\AA 488$ conjugated donkey anti-rabbit IgG, Molecular Probes, 1:200) for $2 \mathrm{~h}$ at RT. Finally, embryos were washed and counterstained with DAPI $(1 \mu \mathrm{g} / \mathrm{ml}$ in PBS, $5 \mathrm{~min})$ and images were obtained as described above.

\section{Acknowledgements}

We thank D. Yao and J. Xia for reagents and other members of the lab for technical support. This work was supported by grants from the "Strategic Priority Research Program" of the Chinese Academy of Sciences (XDA01020307), Ministry of Science and Technology 973 program (2009CB941102) and CAS 100-talent project (X.S.).

\section{References}

BAKKEBO, M., HUSE, K., HILDEN, V.I., FORFANG, L., MYKLEBUST, J.H., SMELAND, E.B. and OKSVOLD, M.P. (2012). SARA is dispensable for functional TGF-beta signaling. FEBS Lett 586: 3367-3372.

BENNETT, D. and ALPHEY, L. (2002). PP1 binds Sara and negatively regulates Dpp signaling in Drosophila melanogaster. Nat Genet 31: 419-423.

BERG, T., ROUNTREE, C.B., LEE, L., ESTRADA, J., SALA, F.G., CHOE, A., VELTMAAT, J.M., DE LANGHE, S., LEE, R., TSUKAMOTO, H. et al., (2007). Fibroblast growth factor 10 is critical for liver growth during embryogenesis and controls hepatoblast survival via beta-catenin activation. Hepatology 46: 1187-1197.

BOKEL, C., SCHWABEDISSEN, A., ENTCHEV, E., RENAUD, O. and GONZALEZGAITAN, M. (2006). Sara endosomes and the maintenance of Dpp signaling levels across mitosis. Science 314: 1135-1139.

CALMONT, A., WANDZIOCH, E., TREMBLAY, K.D., MINOWADA, G., KAESTNER, K.H., MARTIN, G.R. and ZARET, K.S. (2006). An FGF response pathway that mediates hepatic gene induction in embryonic endoderm cells. Dev Cell11:339-348.

CHEN, L., KWONG, M., LU, R., GINZINGER, D., LEE, C., LEUNG, L. and CHAN, J.Y. (2003). Nrf1 is critical for redox balance and survival of liver cells during development. Mol Cell Biol 23: 4673-4686.

CHU, J. and SADLER, K.C. (2009). New school in liver development: lessons from zebrafish. Hepatology 50: 1656-1663.

CURADO, S., ANDERSON, R.M., JUNGBLUT, B., MUMM, J., SCHROETER, E. and STAINIER, D.Y. (2007). Conditional targeted cell ablation in zebrafish: a new tool for regeneration studies. Dev Dyn 236: 1025-1035.

DI GUGLIELMO, G.M., LE ROY, C., GOODFELLOW, A.F. and WRANA, J.L. (2003). Distinct endocytic pathways regulate TGF-beta receptor signalling and turnover. Nat Cell Biol 5: 410-421.

FIELD, H.A., OBER, E.A., ROESER, T. and STAINIER, D.Y. (2003). Formation of the digestive system in zebrafish. I. Liver morphogenesis. Dev Biol 253: 279-290.

GOTO, D., NAKAJIMA, H., MORI, Y., KURASAWA, K., KITAMURA, N. and IWAMOTO, I. (2001). Interaction between Smad anchor for receptor activation and Smad3 is not essential for TGF-beta/Smad3-mediated signaling. Biochem Biophys Res Commun 281: 1100-1105.

JUNG, J., ZHENG, M., GOLDFARB, M. and ZARET, K.S. (1999). Initiation of mammalian liver development from endoderm by fibroblast growth factors. Science 284: 1998-2003.

KUNZMANN, S., WOHLFAHRT, J.G., ITOH, S., ASAO, H., KOMADA, M., AKDIS, C.A., BLASER, K. and SCHMIDT-WEBER, C.B. (2003). SARA and Hgs attenuate susceptibility to TGF-beta1-mediated T cell suppression. FASEB J 17: 194-202.

MCLIN, V.A., RANKIN, S.A. and ZORN, A.M. (2007). Repression of Wnt/beta-catenin signaling in the anterior endoderm is essential for liver and pancreas development. Development 134: 2207-2217.

MICSENYI, A., TAN, X., SNEDDON, T., LUO, J.H., MICHALOPOULOS, G.K. and 
MONGA, S.P. (2004). Beta-catenin is temporally regulated during normal liver development. Gastroenterology 126: 1134-1146.

MONGA, S.P., MONGA, H.K., TAN, X., MULE, K., PEDIADITAKIS, P. and MICHALOPOULOS, G.K. (2003). Beta-catenin antisense studies in embryonic liver cultures: role in proliferation, apoptosis, and lineage specification. Gastroenterology 124: 202-216.

ROSSI, J.M., DUNN, N.R., HOGAN, B.L. and ZARET, K.S. (2001). Distinct mesoderma signals, including BMPs from the septum transversum mesenchyme, are required in combination for hepatogenesis from the endoderm. Genes Dev15: 1998-2009.

RUNYAN, C.E., HAYASHIDA, T., HUBCHAK, S., CURLEY, J.F. and SCHNAPER, H.W. (2009). Role of SARA (SMAD anchor for receptor activation) in maintenance of epithelial cell phenotype. J Biol Chem 284: 25181-25189.

RUNYAN, C.E., SCHNAPER, H.W. and PONCELET, A.C. (2005). The role of internalization in transforming growth factor beta1-induced Smad2 association with Smad anchor for receptor activation (SARA) and Smad2-dependent signaling in human mesangial cells. J Biol Chem 280: 8300-8308.

SANCHEZ, A. and FABREGAT, I. (2010). Growth factor- and cytokine-driven pathways governing liver stemness and differentiation. World J Gastroentero/16:5148-5161.

SEKHON, S.S., TAN, X., MICSENYI, A., BOWEN, W.C. and MONGA, S.P. (2004). Fibroblast growth factor enriches the embryonic liver cultures for hepatic progenitors. Am J Pathol 164: 2229-2240.

SFLOMOS, G., KOSTARAS, E., PANOPOULOU, E., PAPPAS, N., KYRKOU, A., POLITOU, A.S., FOTSIS, T. and MURPHY, C. (2011). ERBIN is a new SARAinteracting protein: competition between SARA and SMAD2 and SMAD3 for binding to ERBIN. J Cell Sci 124: 3209-3222.

SHIN, D., SHIN, C.H., TUCKER, J., OBER, E.A., RENTZSCH, F., POSS, K.D., HAMMERSCHMIDT, M., MULLINS, M.C. and STAINIER, D.Y. (2007). Bmp and Fgf signaling are essential for liver specification in zebrafish. Development 134: 2041-2050.

SI-TAYEB, K., LEMAIGRE, F.P. and DUNCAN, S.A. (2010). Organogenesis and development of the liver. Dev Cell 18: 175-189

SUKSAWEANG, S., LIN, C.M., JIANG, T.X., HUGHES, M.W., WIDELITZ, R.B. and CHUONG, C.M. (2004). Morphogenesis of chicken liver: identification of localized growth zones and the role of beta-catenin/Wnt in size regulation. Dev Biol 266: 109-122.

TAN, X., YUAN, Y., ZENG, G., APTE, U., THOMPSON, M.D., CIEPLY, B., STOLZ D.B., MICHALOPOULOS, G.K., KAESTNER, K.H. and MONGA, S.P. (2008). Beta-catenin deletion in hepatoblasts disrupts hepatic morphogenesis and survival during mouse development. Hepatology 47: 1667-1679.

TAO, T. and PENG, J. (2009). Liver development in zebrafish (Danio rerio). J Genet Genomics 36: 325-334.

TSUKAZAKI, T., CHIANG, T.A., DAVISON, A.F., ATTISANO, L. and WRANA, J.L. (1998). SARA, a FYVE domain protein that recruits Smad2 to the TGFbeta receptor. Cell 95: 779-791.

WEINSTEIN, M., MONGA, S.P., LIU, Y., BRODIE, S.G., TANG, Y., LI, C., MISHRA L. and DENG, C.X. (2001). Smad proteins and hepatocyte growth factor control parallel regulatory pathways that converge on beta1-integrin to promote normal liver development. Mol Cell Biol 21: 5122-5131.

WESTERFIELD, M. (1995). The Zebrafish Book. A Guide for the Laboratory Use of Zebrafish (Danio rerio). Eugene: University of Oregon.

XU, L., YIN, W., XIA, J., PENG, M., LI, S., LIN, S., PEI, D. and SHU, X. (2012). An antiapoptotic role of sorting nexin 7 is required for liver development in zebrafish. Hepatology 55: 1985-1993.

YANAI, M., TATSUMI, N., HASUNUMA, N., KATSU, K., ENDO, F. and YOKOUCHI Y. (2008). FGF signaling segregates biliary cell-lineage from chick hepatoblasts cooperatively with BMP4 and ECM components in vitro. Dev Dyn 237: 1268-1283.

ZHANG, W., YATSKIEVYCH, T.A., BAKER, R.K. and ANTIN, P.B. (2004). Regulation of Hex gene expression and initial stages of avian hepatogenesis by Bmp and Fgf signaling. Dev Biol 268: 312-326. 


\section{Further Related Reading, published previously in the Int. J. Dev. Biol.}

Zebrafish embryo, a tool to study tumor angiogenesis

Chiara Tobia, Giulia De Sena and Marco Presta

Int. J. Dev. Biol. (2011) 55: 505-509

The role of angiogenic growth factors in organogenesis

Enrico Crivellato

Int. J. Dev. Biol. (2011) 55: 365-375

Hematopoietic development in the zebrafish

Elizabeth J. Paik and Leonard I. Zon

Int. J. Dev. Biol. (2010) 54: 1127-1137

Spatiotemporal expression of the creatine metabolism related genes agat, gamt and ct1 during zebrafish embryogenesis

Lifeng Wang, Ying Zhang, Ming Shao and Hongwei Zhang

Int. J. Dev. Biol. (2007) 51: 247-253

C/EBPalpha and C/EBPbeta are markers of early liver development

Adam Westmacott, Zoë D. Burke, Guillermo Oliver, Jonathan M.W. Slack and David Tosh Int. J. Dev. Biol. (2006) 50: 653-657

5 yr ISI Impact Factor $(2011)=2.959$
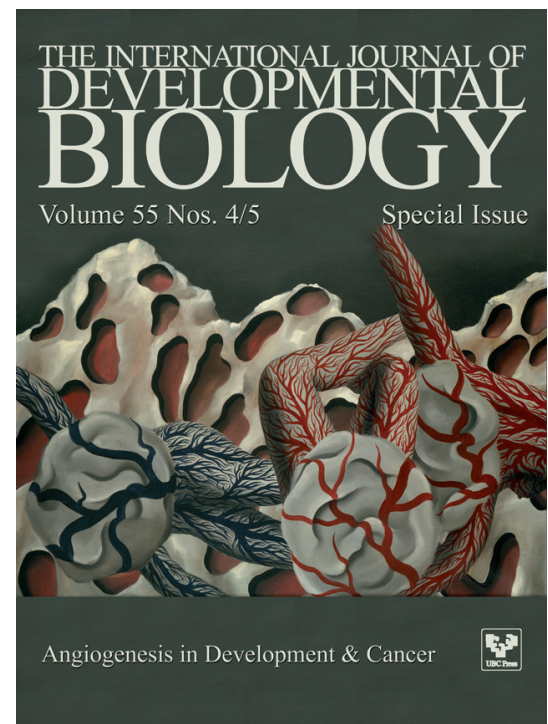

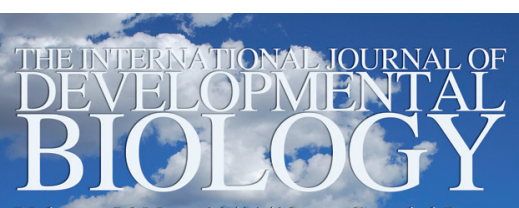

Volume 56 Nos. 10/11/12 - Special Issue

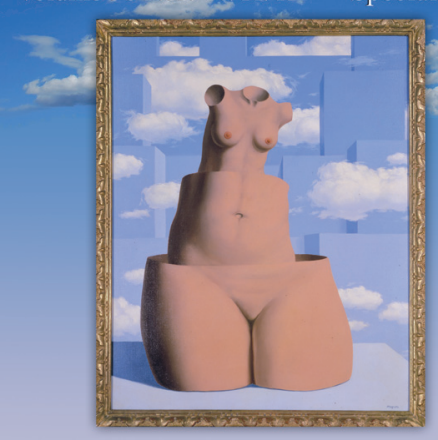

Female Germ Cells in Development \& Tumors
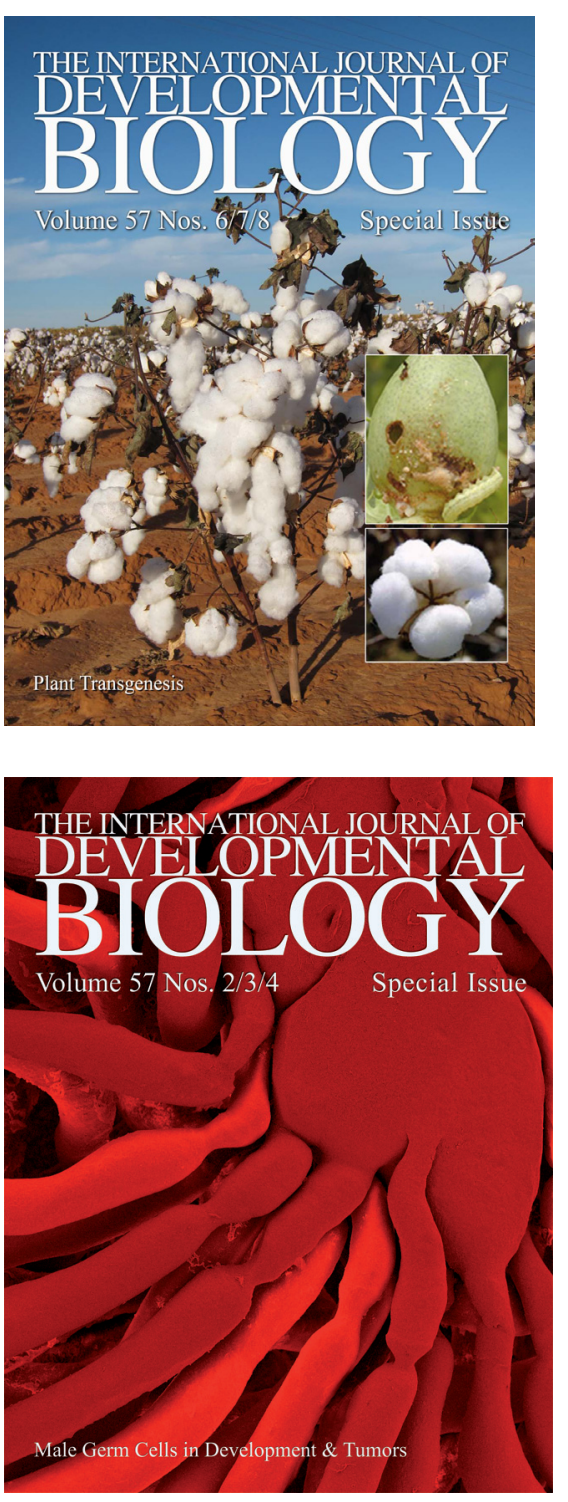